\title{
Targeted Top-down Mass Spectrometry for the Characterization and Tissue-specific Functional Discovery of Crustacean Hyperglycemic Hormones (CHH) and CHH Precursor-Related Peptides in Response to Low pH Stress
}

Yang Liu ${ }^{a}$, Gongyu Li ${ }^{b}$, Lingjun Li*a,b

${ }^{a}$ Department of Chemistry, 1101 University Avenue, University of Wisconsin, Madison, WI, 53706

${ }^{b}$ School of Pharmacy, 777 Highland Avenue, University of Wisconsin, Madison, WI, 53705

*Address reprint requests to:

Dr. Lingjun Li, School of Pharmacy \& Department of Chemistry, University of Wisconsin, 5125 Rennebohm Hall, 777 Highland Avenue, Madison, WI 53705-2222

E-mail: lingjun.li@wisc.edu. Phone: (608)265-8491, Fax: (608)262-5345. 


\section{Table of Contents}

Methods. Chemicals and materials

Methods. Animals, Low pH Stress Experiment and Tissue Extraction

Methods. High pH HPLC Fractionation

Methods. Reductive Dimethylation Labeling

Figure S1. Top-down HCD MS/MS spectra of non-reduced (A), DTT-reduced (B) Cas-SG-CHH and non-reduced (C), DTT-reduced (D) Cas-PO-CHH. The spectra in A, B and D were deconvoluted using Xtract to decrease the complexity. For both $\mathrm{CHH}$ isoforms, more b/y ions were identified after DTT treatment, which provide higher coverage in top-down sequencing analysis.

Figure S2. Sequence coverage map of Cas-SG-CHH (A) (B) and Cas-PO-CHH (C) (D) acquired using Fusion Lumos Orbitrap mass spectrometer. Red cells with " + " represented the amino acid residue was identified. Green cells with "-" represented that there was no sufficient fragmentation information for the AA residue identification. Panel $A$ and $C$ were obtained using HCD fragmentation. Panel B and D were resulted from hybrid fragmentation, EThcD. Energy level expression can be interpreted as ETD reaction time/HCD collision energy.

Figure S3. Top-down MS/MS HCD spectra of reductive formaldehyde labeled Cas-SG-CHH. Light and heavy labeled samples in the quantitative peak pairs were represented by red and blue circles, respectively. Labeling dynamic range has been tested from 1:0.1 to 1:10. 


\section{Methods Supporting Information}

\section{Chemical and Materials}

Methanol (MeOH), acetonitrile ( $\mathrm{ACN}$ ), formic acid (FA), ammonium bicarbonate, ammonium formate and glacial acetic acid were purchased from Fisher Scientific (Pittsburgh, PA). Borane pyridine, formaldehyde, and deuterium formaldehyde were from Sigma-Aldrich (St. Louis, MO). Acidified methanol was prepared as $90 \%$ methanol, $9 \%$ water, and $1 \%$ glacial acetic acid (v/v/v). Double distilled water (Millipore filtration system, Burlington, MA) or Fisher HPLC grade water was used in this study.

\section{Animals, Low pH Stress Experiment and Tissue Extraction}

The Atlantic blue crabs, Callinectes sapidus, female, were purchased from Midway Asian Market (Madison, WI). In lab, the crabs were maintained in automated recirculating artificial seawater (30 ppt salinity, 12h:12h light/dark cycle). Animals were starved for at least one week to recover from transportation stress. Active crabs at similar body size were selected for quantitative analysis. The low $\mathrm{pH}$ stress experiment setup was described in detail in a previous study ${ }^{16}$. To be brief, control and stress animals were separated into two 10 -gallon tanks. Initial $\mathrm{pH}$ level was maintained at 8.3 (normal pH for seawater) using marine buffer (Seachem Laboratories, Madison, $\mathrm{GA}$ ). $\mathrm{CO}_{2}$ gas was introduced into the tank to acidify the water. $\mathrm{A} \mathrm{pH}$ sensor (American Marine Pinpoint pH Monitor) and a dissolved oxygen (D.O.) sensor (American Marine Pinpoint Oxygen Monitor) were used to track the $\mathrm{pH}$ and D.O. level. The low $\mathrm{pH}$ stress experiment in this work was performed at a moderate $\mathrm{pH}$ level (7.6-7.8) and air saturation 50\%$60 \%$. The crab was incubated in the stable acidified environment for 2 hours.

For both characterization and quantitation experiments, crabs were then cold-anesthetized on ice for 15-20 min before being sacrificed. Pericardial organs and sinus glands were collected in chilled physiological saline (Composition: $440 \mathrm{mM} \mathrm{NaCl}, 11 \mathrm{mM} \mathrm{KCl}, 13 \mathrm{mM} \mathrm{CaCl}$, $26 \mathrm{mM}$ $\mathrm{MgCl}$, and $10 \mathrm{mM}$ HEPES (4-(2-hydroxyethyl)-1-piperazineethanesulfonic acid) acid; $\mathrm{pH} 7.4$, adjusted with $\mathrm{NaOH}$ ). The detailed dissection procedure was carried out as described previously. ${ }^{57}$ Tissues were stored in acidified methanol. They were manually homogenized and extracted using acidified methanol. Large proteins were removed by centrifugation (16 $100 \mathrm{xg}$ for $10 \mathrm{~min}$ ) and the supernatant containing neuropeptides were combined from repeated washes. Extracted neuropeptide solution was dried down and subjected to further analysis.

\section{High pH HPLC Fractionation}

$100 \mu$ of crude tissue extract was loaded to a Phenomenex Gemini $C_{18}$ column (2.1mm i.d., 150 $\mathrm{mm}$ length, $5 \mu \mathrm{m}$ particle size; Torrance, $\mathrm{CA}$ ) and the offline separation was performed with a 
Waters Alliance HPLC system (Milford, MA). The mobile phases were prepared as following: solution $\mathrm{A}\left(\mathrm{H}_{2} \mathrm{O}\right.$ containing $10 \mathrm{mM}$ ammonium formate, $\left.\mathrm{pH}=10\right)$ and solution $\mathrm{B}(90 \% \mathrm{ACN}$ containing $10 \mathrm{mM}$ ammonium formate, $\mathrm{pH}=10$ ). $\mathrm{pH}$ level was adjusted with ammonium hydroxide. The flow rate was $0.2 \mathrm{ml} / \mathrm{min}$. The separation was performed under a $95 \mathrm{~min}$ gradient: mobile phase B was at $1 \%$ for the first $5 \mathrm{~min}$ and increased to $40 \%$ at $50 \mathrm{~min}, 60 \%$ at $54 \mathrm{~min}, 70 \%$ at $58 \mathrm{~min}, 100 \%$ at $59 \mathrm{~min}$ (remaining for $15 \mathrm{~min}$ ), then dropped back to $1 \%$ at 75 min. Fractionation was collected every 1 min with a Rainin Dynamax FC-4 fraction collector. Collected fractionations were lyophilized for further steps.

\section{Reductive Dimethylation Labeling}

Both control and stressed tissue extracts were resuspended in $10 \mu \mathrm{l} 90 \%$ ACN in water and labeled with formaldehyde- $\mathrm{H}_{2}(1 \% \mathrm{v} / \mathrm{v})$ and formaldehyde- $\mathrm{D}_{2}(1 \% \mathrm{v} / \mathrm{v})$, respectively. Borane pyridine $(30 \mathrm{mM})$ was added to the sample, followed by 1-hour incubation at room temperature. $100 \mathrm{mM}$ ammonium bicarbonate was added to quench the reaction. Two samples (control and stressed) were mixed at equal volume, dried down and resuspended in $10 \mu \mathrm{l} 0.1 \%$ FA in water. $\mathrm{C}_{18}$ Ziptip pipette tips (Merck Millipore Ltd., Tullagreen, Carrightwohill, Co. Cork IRL) was used to desalt the samples before MS analysis. 


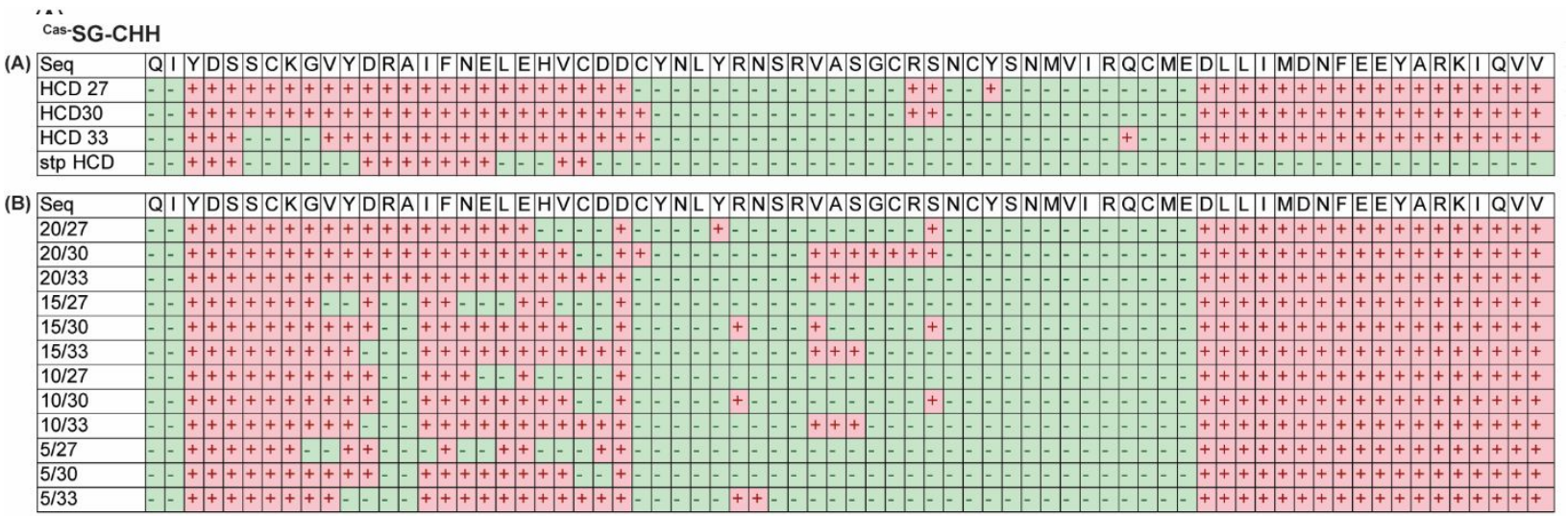

Cas-PO-CHH

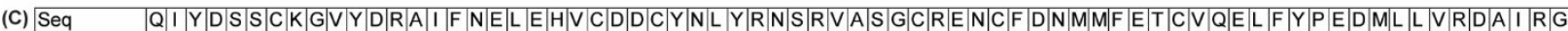

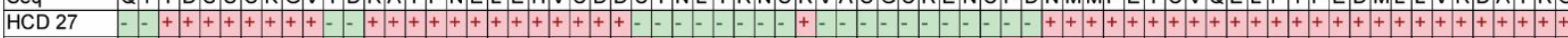

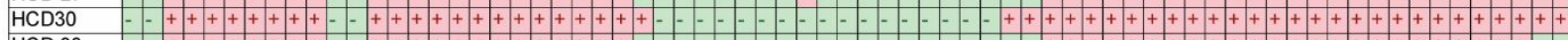

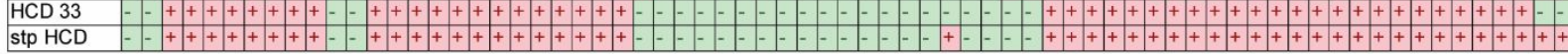

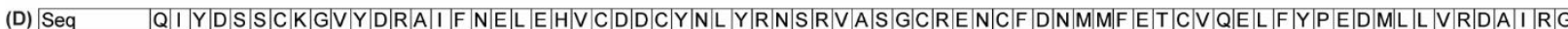
$20 / 27 \quad--+++++++++++--+++--+++++-6++++++++++++++++++++++++++++++++++++--++++++$

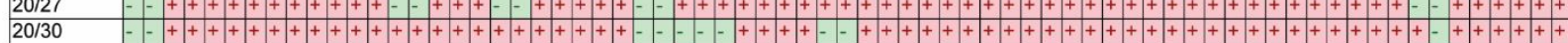

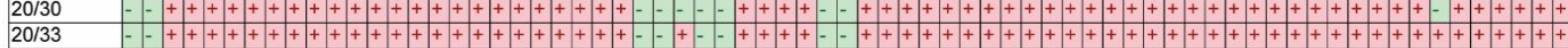

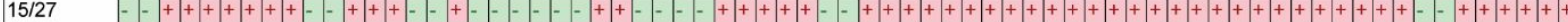

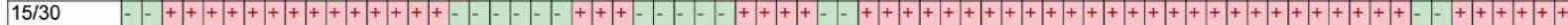

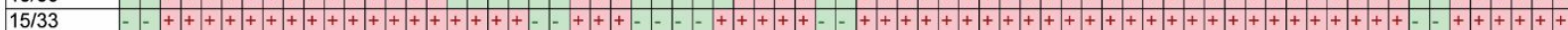
$10 / 27 \quad-.++++++++++++++\ldots-\ldots++-6+++++-++++++++++++-++++++++++++-+++++$

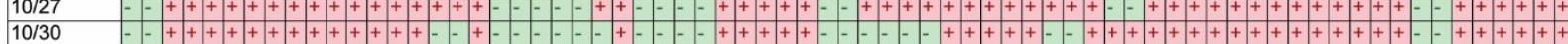

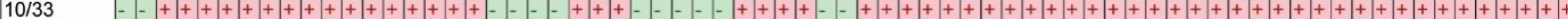
$5 / 27 \quad--+++++++++++--+++------+----+++++++++++--+++--+++++++++++--+++---+--++$

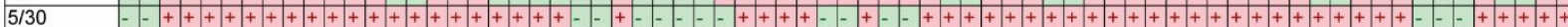

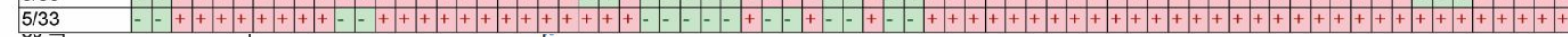
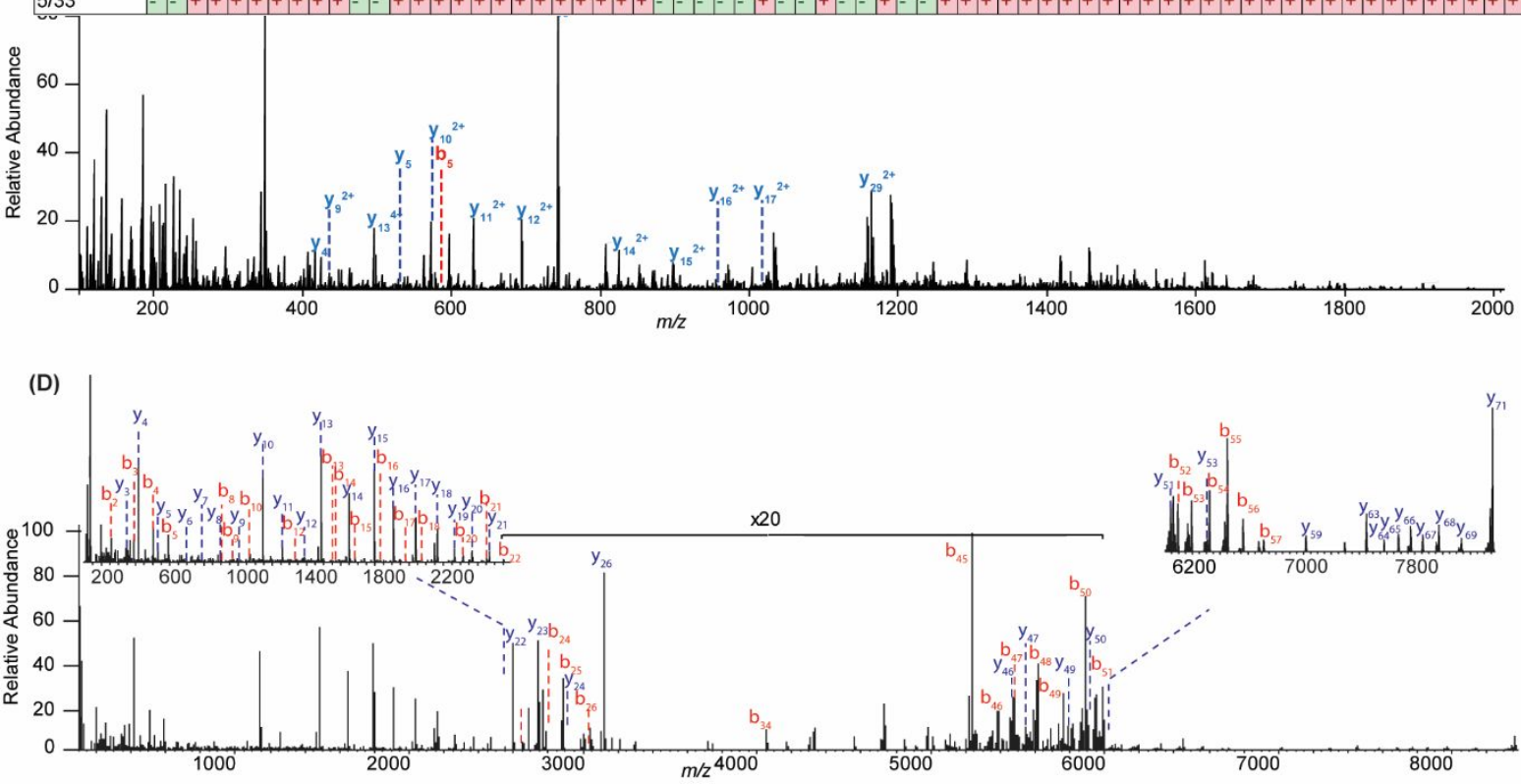

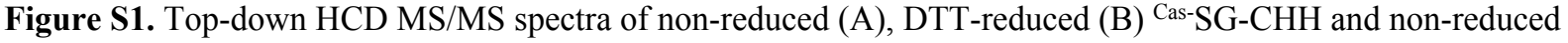
(C), DTT-reduced (D) Cas-PO-CHH. The spectra in (A), (B) and (D) were deconvoluted using Xtract to decrease the complexity. For both $\mathrm{CHH}$ isoforms, more b/y ions were identified after DTT treatment, which provided higher coverage in top-down sequencing analysis.

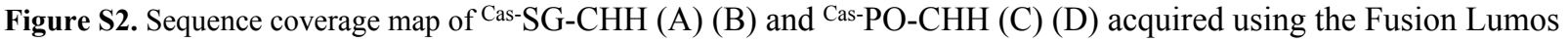
Orbitrap mass spectrometer. Red cells with "+" represented the amino acid residue was identified. Green cells with "-" represented that there was no sufficient fragmentation information for the AA residue identification. Panel A and $\mathrm{C}$ were obtained using HCD fragmentation. Panel B and D were resulted from hybrid fragmentation, EThcD. Energy level expression can be interpreted as ETD reaction time/HCD collision energy. 


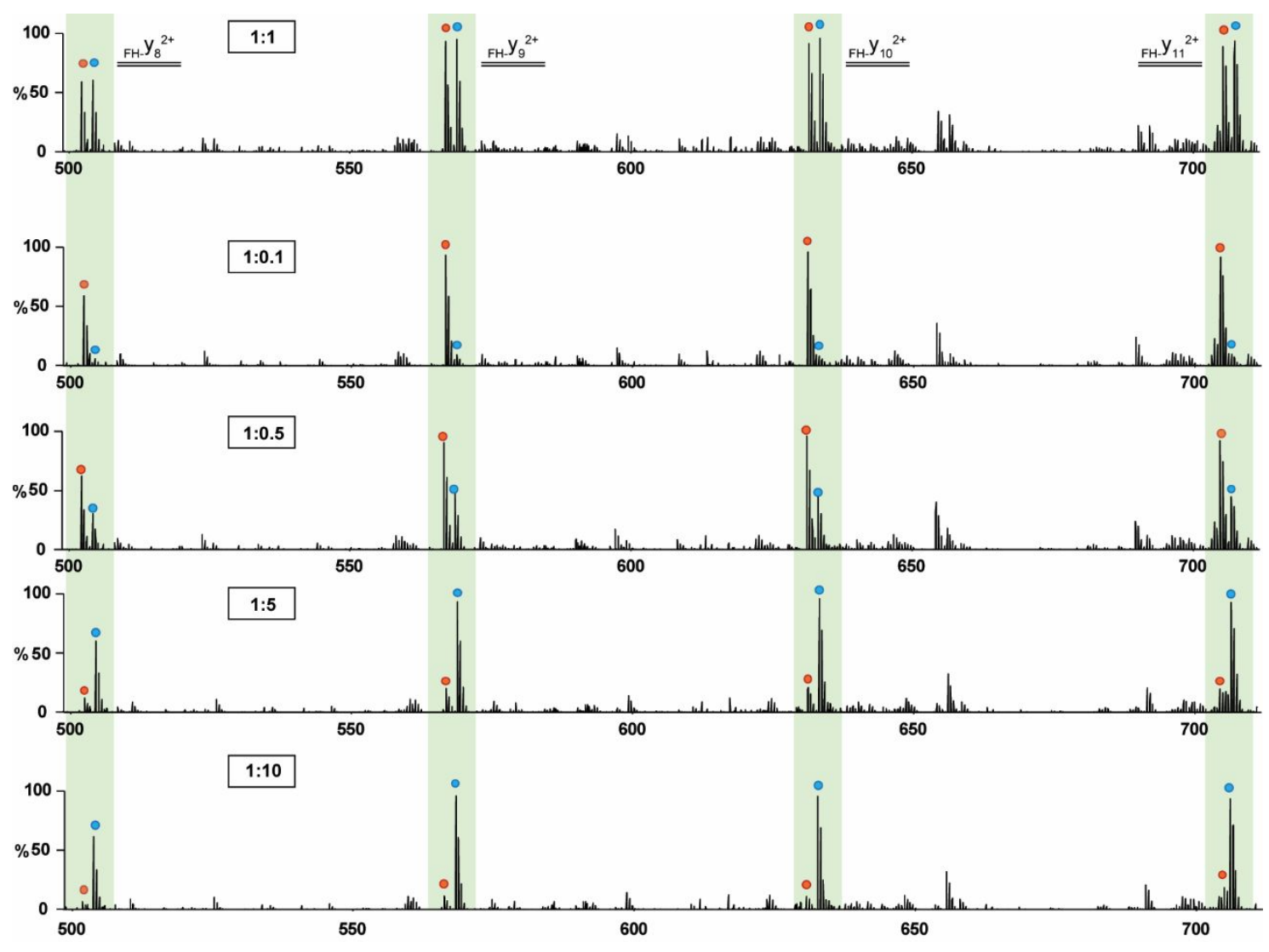

Figure S3. Top-down MS/MS HCD spectra of reductive formaldehyde labeled Cas-SG-CHH. Light and heavy labeled samples in the quantitative peak pairs were represented by red and blue circles, respectively. Labeling dynamic range has been tested from 1:0.1 to 1:10. 